\title{
VEGF-B inhibits apoptosis via VEGFR-1- mediated suppression of the expression of BH3-only protein genes in mice and rats
}

\author{
Yang Li, ${ }^{1}$ Fan Zhang, ${ }^{1}$ Nobuo Nagai, ${ }^{2}$ Zhongshu Tang, ${ }^{1}$ Shuihua Zhang, ${ }^{1}$ Pierre Scotney, ${ }^{3}$ \\ Johan Lennartsson, ${ }^{4}$ Chaoyong Zhu, ${ }^{1}$ Yi Qu, ${ }^{1}$ Changge Fang, ${ }^{1}$ Jianyuan Hua, ${ }^{1}$ Osamu Matsuo, ${ }^{2}$ \\ Guo-Hua Fong, ${ }^{5}$ Hao Ding, ${ }^{6}$ Yihai Cao, ${ }^{7}$ Kevin G. Becker, ${ }^{8}$ Andrew Nash, ${ }^{3}$ \\ Carl-Henrik Heldin, ${ }^{4}$ and Xuri Li ${ }^{1}$
}

${ }^{1}$ National Eye Institute, NIH, Porter Neuroscience Research Center, Bethesda, Maryland, USA. 2Department of Physiology, Kinki University School of Medicine, Osakasayama, Osaka, Japan. ${ }^{3}$ CSL Limited, Parkville, Victoria, Australia. ${ }^{4}$ Ludwig Institute for Cancer Research, Uppsala University, Uppsala, Sweden. ${ }^{5}$ Center for Vascular Biology, University of Connecticut Health Center, Farmington, Connecticut, USA. ${ }^{6}$ Department of Biochemistry and Medical Genetics, University of Manitoba, Winnipeg, Manitoba, Canada. ${ }^{7}$ Department of Microbiology, Tumor and Cell Biology, Karolinska Institute, Stockholm, Sweden. ${ }^{8 T R I A D}$ Technology Center, National Institute on Aging, NIH, Baltimore, Maryland, USA.

\begin{abstract}
Despite its early discovery and high sequence homology to the other VEGF family members, the biological functions of VEGF-B remain poorly understood. We revealed here a novel function for VEGF-B as a potent inhibitor of apoptosis. Using gene expression profiling of mouse primary aortic smooth muscle cells, and confirming the results by real-time PCR using mouse and rat cell lines, we showed that VEGF-B inhibited the expression of genes encoding the proapoptotic $\mathrm{BH}$-only proteins and other apoptosis- and cell death-related proteins, including p53 and members of the caspase family, via activation of VEGFR-1. Consistent with this, VEGF-B treatment rescued neurons from apoptosis in the retina and brain in mouse models of ocular neurodegenerative disorders and stroke, respectively. Interestingly, VEGF-B treatment at the dose effective for neuronal survival did not cause retinal neovascularization, suggesting that VEGF-B is the first member of the VEGF family that has a potent antiapoptotic effect while lacking a general angiogenic activity. These findings indicate that VEGF-B may potentially offer a new therapeutic option for the treatment of neurodegenerative diseases.
\end{abstract}

\section{Introduction}

The VEGF family incorporates 5 structurally related ligands that bind differentially to 3 receptor tyrosine kinases (VEGFR-1,-2, and -3) and the semaphorin receptors neuropilin 1 (NP-1) and NP-2. Among the 5 VEGF family members, VEGF is the prototypical angiogenic factor, with a potent and universal angiogenic effect in most physiological and pathological conditions (1-3). The placenta growth factor (PlGF) is believed to play important roles in pathological angiogenesis (4), while VEGF-C and VEGF-D are mainly involved in lymphangiogenesis (5). VEGF has recently been shown to be a potent neuroprotective factor $(6,7)$. However, the therapeutic potential of its neuroprotective effect is limited by its potent and general activity in inducing undesired angiogenesis and blood vessel leakage, which is highly detrimental in most conditions.

Compared with the other members of the VEGF family, VEGF-B has received much less attention, and its biological function remains debatable $(8,9)$. VEGF-B shares a high degree of sequence homology with VEGF and PlGF. It binds to the tyrosine kinase VEGFR-1 and its coreceptor NP-1 $(10,11)$. VEGF-B is highly expressed in most tissues and organs $(12,13)$. VEGF-B in most conditions seems to be redundant or inert. In contrast to VEGF-

Nonstandard abbreviations used: $\mathrm{CNV}$, choroidal neovascularization; ECD, extracellular domain; IB4, isolectin B4; LDH, lactate dehydrogenase; MCA, middle cerebral artery; NP-1, neuropilin 1; ONC, optic nerve crush; PlGF, placenta growth factor; RGC, retinal ganglion cell.

Conflict of interest: A. Nash and P. Scotney receive income as employees of CSL Ltd., and A. Nash holds stock options. The rest of the authors have declared that no conflict of interest exists.

Citation for this article: J. Clin. Invest. 118:913-923 (2008). doi:10.1172/JCI33673. deficient mice $(14,15)$, VEGF-B-deficient mice are healthy and fertile, with apparently normal developmental and physiological angiogenesis $(16,17)$. In contrast to VEGF and PlGF, VEGF-B is not required for neovessel formation in proliferative retinopathy (18) and blood vessel remodeling in pulmonary hypertension (19). Adenoviral VEGF-B gene transfer in the hind limb (20) and carotid artery (21) did not induce any angiogenic response, while the other VEGF family members did. Moreover, numerous studies have shown that VEGF-B has no effect on blood vessel permeability (19, 22-24), in contrast to the other VEGF family members.

Current functional analysis of VEGF-B has led to inconsistent and controversial results. While one study reported smaller heart and abnormal coronary artery vasculature caused by VEGF-B deficiency in mice (17), this was not observed in another independent study (16). Several reports have shown an angiogenic effect of VEGF-B (24-26), whereas other studies have observed the lack of angiogenic activity of VEGF-B in different model systems $(16,18$, $20,21,27)$. VEGF-B was reported to play a role in pulmonary hypertension (28); however, this was not observed in another independent study (19). In summary, due to various reasons including the distinct gene-targeting approaches used, the different genetic background of the animals in different studies, and experimental variations among different research groups, the reports published thus far with regard to the function of VEGF-B have been inconsistent. Further studies on VEGF-B are therefore especially warranted.

In this study, to determine the biological function of VEGF-B, we performed a genome-wide gene expression profiling assay. We validated our findings in multiple cell lines and animal models. We report here that VEGF-B is a potent apoptosis inhibitor by sup- 
Table 1

Apoptotic/cell death-related genes downregulated by VEGF-B 167

\begin{tabular}{|c|c|c|}
\hline Gene symbol & GenBank accession no. & Fold downregulated \\
\hline$B m f$ & AK040622 & 9.60 \\
\hline Trp53inp1 & NM_021897 & 7.90 \\
\hline Den & NM_007833 & 7.34 \\
\hline Angpt2 & NM_007426 & 4.98 \\
\hline Sesn1 & BC055753 & 4.80 \\
\hline Txnip & AK004653 & 4.62 \\
\hline Cables1 & NM_022021 & 4.55 \\
\hline Aox1 & NM_009676 & 3.98 \\
\hline Abtb1 & NM_030251 & 3.69 \\
\hline Ptpn13 & NM_011204 & 3.35 \\
\hline Map3k7ip2 & NM_138667 & 3.10 \\
\hline Noxa & NM_021451 & 2.95 \\
\hline Scn2a1 & AK089150 & 2.80 \\
\hline Mbd4 & NM_010774 & 2.75 \\
\hline lkbkg & AK042138 & 2.55 \\
\hline Casp2 & NM_007610 & 2.42 \\
\hline Prkdc & NM_011159 & 2.40 \\
\hline Aim1 & NM_172393 & 2.26 \\
\hline$R b / 2$ & NM_011250 & 2.30 \\
\hline Map3k1 & NM_011945 & 2.23 \\
\hline Aatk & NM_007377 & 2.20 \\
\hline Plag/1 & BCōo65150 & 2.19 \\
\hline Tlr2 & NM_011905 & 2.19 \\
\hline Adrb2 & NM_007420 & 2.16 \\
\hline Cradd & NM_009950 & 2.12 \\
\hline$A r$ & NM_013476 & 2.11 \\
\hline Dedd2 & AK010701 & 2.10 \\
\hline $\operatorname{ltg} b 3 b p$ & AK088352 & 2.10 \\
\hline Thap3 & NM_175152 & 2.10 \\
\hline Ifih1 & NM_027835 & 2.00 \\
\hline
\end{tabular}

pressing the expression of the BH3-only protein and other apoptotic/cell death-reælated genes via VEGFR-1. VEGF-B treatment rescues neurons from apoptosis in both the retina and brain without causing undesired angiogenesis. VEGF-B therefore appears to be the first member of the VEGF family that has been observed to have a potent antiapoptotic effect but lack general angiogenic activity. VEGF-B thus may potentially offer a new therapeutic option for the treatment of neurodegenerative diseases.

\section{Results}

Genome-wide gene profiling reveals that VEGF-B inbibits the expression of numerous apoptotic/cell death-related genes. To determine the biological function of VEGF-B, we performed a genome-wide gene expression profiling assay to identify the genes regulated by VEGF-B. We treated mouse primary aortic SMCs with human recombinant VEGF- $B_{167}$ protein in a hypoxic condition ( $1 \%$ oxygen), because these cells express VEGFR-1 and VEGFR-1 is upregulated in hypoxia (data not shown). We performed the expression profiling analysis using the whole mouse genome microarray containing 41,534 mouse genes and transcripts. Results revealed that VEGF-B treatment significantly downregulated the expression of about 600 genes by more than 2 -fold. Among them, the genes downregulated to the greatest extent fall into the apoptosis/cell deathrelated pathways (Table 1). The 3 most downregulated genes were the ones critically involved in apoptosis: $\operatorname{Bmf}(29)$ (about 10-fold downregulated), TrP53inp1 (30) (about 8-fold downregulated), and
DCN (31) (about 7-fold downregulated). Thus, the genes that are downregulated to the greatest extent by VEGF-B are the ones critically involved in the apoptosis/cell death-related pathways.

VEGF-B inhibits apoptotic gene expression in multiple cells and cell lines. We next used 5 different types of cells/cell lines to verify the microarray data. We treated the rat retinal ganglion cell-derived (RGC-derived) cell line RGC5, the immortalized rat retinal pericyte cell line TR-rPCT, the immortalized rat retinal Müller cell line TRMUL, the immortalized rat retinal endothelial cell line TR-iBRB, and mouse primary aortic SMCs (mSMCs) with VEGF-B 167 (100 $\mathrm{ng} / \mathrm{ml}$ ) for 6 hours in either normoxic or hypoxic (1\% oxygen) conditions, and the expression of the apoptotic/cell death-related genes was investigated by real-time PCR assay. VEGFR-1 expression was detected by real-time PCR assay in all the 5 different types of cells (data not shown). VEGF-B significantly inhibited the expression of many apoptotic/cell death-related genes, consistent with the microarray data. Among these genes, the expression of most of the BH3-only protein genes (Bmf, Hrk, Puma, Noxa [Pmaip1], $\mathrm{Bad}, \mathrm{Bid}, \mathrm{Bik}$ ) was inhibited by VEGF-B in the different types of cells (Figure 1, A-E). The $\mathrm{BH}$-only protein genes are therefore the major target genes suppressed by VEGF-B. VEGF-B also inhibited the expression of p53, the caspases (Casp2, Casp8, Casp9, Casp12), and other apoptotic/cell death-related genes (Bak, Bax, Bcl2l11, TNF- $\alpha, D c n$, Olr1, etc.; Figure 1, A-E). Thus, VEGF-B inhibited the expression of the $\mathrm{BH} 3$-only protein and other apoptotic/cell death-related genes in all of the 5 types of cells/cell lines investigated, indicating a general role of VEGF-B as an apoptosis inhibitor in different types of cells.

VEGF-B inhibits oxidative stress-, serum deprivation-, and Bmf-induced apoptosis in cultured cells. We next used different approaches to test whether VEGF-B could inhibit apoptosis in the RGC5 cells. We first treated the RGC5 cells with hydrogen peroxide $\left(\mathrm{H}_{2} \mathrm{O}_{2}\right)$, which is known to induce oxidative stress-induced apoptosis, and investigated the apoptosis status using the TUNEL assay. VEGF-B 167 treatment $(100 \mathrm{ng} / \mathrm{ml})$ significantly decreased the $\mathrm{H}_{2} \mathrm{O}_{2}$-induced apoptosis in the RGC5 cells by about 50\% (Figure 2, A-D; $n=3$, $P<0.01)$. We next tested whether VEGF-B could inhibit the serum deprivation-induced cell death in the RGC5 cells. We cultured the cells in serum-free medium and treated the cells with recombinant proteins of human VEGF-B $167(100 \mathrm{ng} / \mathrm{ml}), \operatorname{VEGF}(25$ and $100 \mathrm{ng} /$ $\mathrm{ml}$ ), and PlGF (25 and $100 \mathrm{ng} / \mathrm{ml}$ ), and the viability of the cells was measured at different time point using the MTT assay. VEGF-B 167 treatment significantly increased cell survival as potently as $10 \%$ FCS at days 1 and 2 (Figure 2E; $n=3, P<0.01$ ). The survival effect of VEGF-B ${ }_{167}$ on the RGC5 cells was slightly greater in a hypoxic condition (data not shown). In contrast, PlGF, another VEGFR-1 ligand, did not show such an effect (Figure 2F). VEGF had only a weak survival effect on the RGC5 cells (Figure 2G; $n=3, P<0.05$ ).

$\mathrm{Bmf}$ is an essential apoptosis inducer in response to cellular stresses (29). Overexpression of Bmfled to cellular apoptosis within 24 hours (29). The potent inhibitory effect of VEGF-B on the expression of Bmf indicates that VEGF-B may inhibit Bmf-mediated apoptosis. To test this, we overexpressed the mouse $B m f$ gene in the rat RGC5 cells and determined the apoptosis rate and the expression of Bmf level in the cells with or without VEGF-B treatment. Bmf overexpression significantly induced cellular apoptosis in the RGC5 cells (Figure 2, H, I, and $\mathrm{K} ; n=3, P<0.01$ ). VEGF-B 167 treatment $(100 \mathrm{ng} / \mathrm{ml}$ for 48 hours) inhibited the Bmf-induced apoptosis by about $40 \%$ (Figure 2 , J and $\mathrm{K} ; n=3, P<0.01$ ). Further, VEGF-B treatment inhibited the expression of both the endog- 

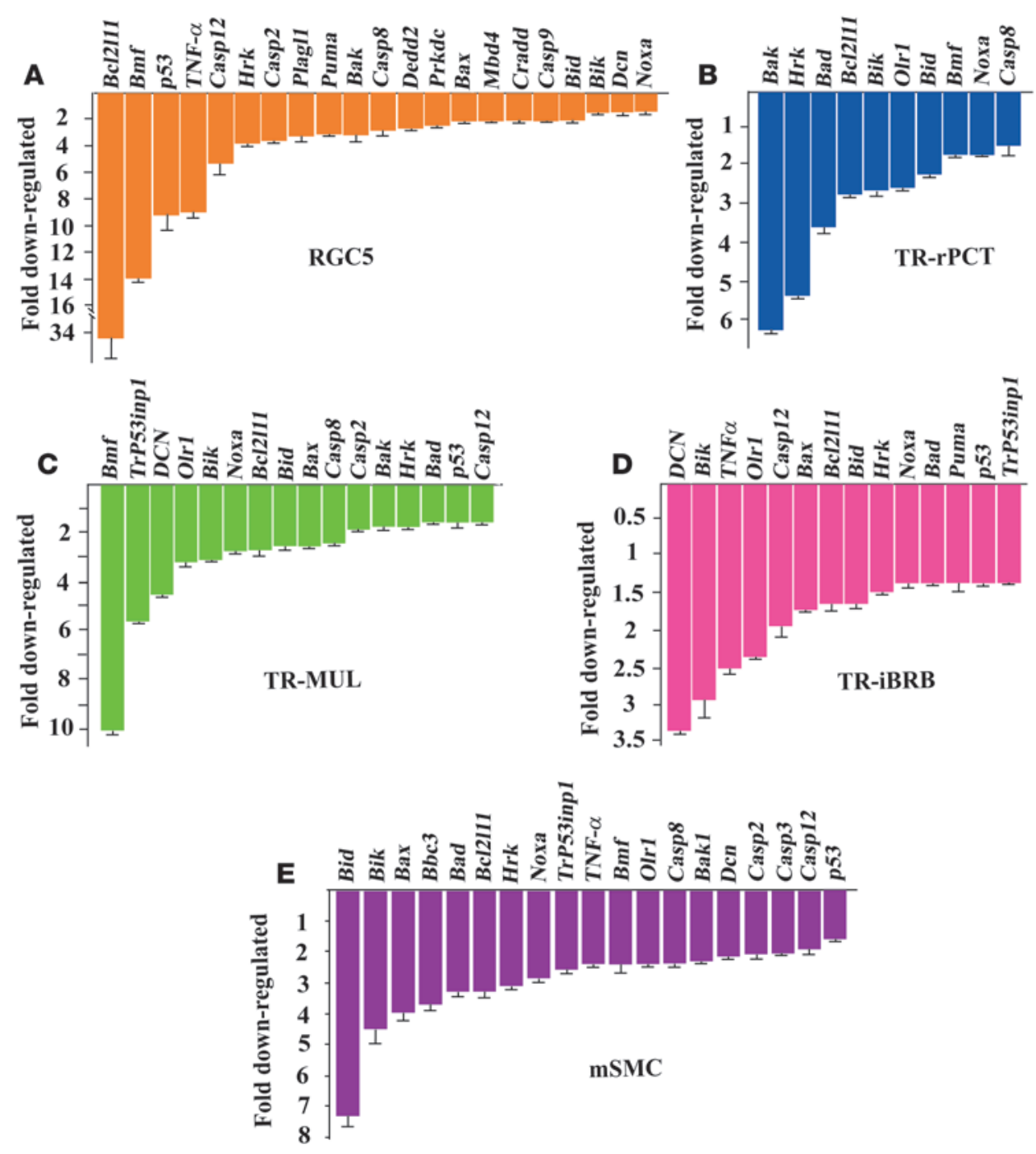

\section{Figure 1}

VEGF-B ${ }_{167}$ inhibits the expression of the BH3-only protein and other apoptotic/cell death-related genes in multiple cell lines. (A) VEGF-B ${ }_{167}$ treatment inhibited the expression of the BH3-only protein (Bmf, Hrk, Puma, Bid, Bik, Noxa) and other apoptotic/cell death-related genes in the rat RGC-derived cell line RGC5 according to real-time PCR assay. (B) VEGF-B ${ }_{167}$ treatment inhibited the expression of the $\mathrm{BH} 3$-only protein (Hrk, Bik, Bid, Bmf, Noxa) and other apoptotic/cell death-related genes in the immortalized rat retinal pericyte cell line TR-rPCT. (C) VEGF-B ${ }_{167}$ treatment inhibited the expression of the $\mathrm{BH}$-only protein (Bmf, Bik, Noxa, Bid, Hrk) and other apoptotic/cell death-related genes in the immortalized rat retinal Müller cell line TR-MUL. (D) VEGF-B ${ }_{167}$ treatment inhibited the expression of the BH3-only protein (Bik, Bid, Hrk, Noxa, Puma) and other apoptotic/cell death-related genes in the immortalized rat retinal endothelial cell line TR-iBRB. (E) VEGF-B ${ }_{167}$ treatment inhibited the expression of the BH3only protein (Bid, Bik, Bbc3, Bad, Hrk, Noxa, Bmf) and other apoptotic/ cell death-related genes in mouse primary aortic smooth muscle cells (mSMC). enous rat and the exogenous mouse $B m f$ genes (Figure $2 \mathrm{~L} ; n=3$, $P<0.01)$. Thus, VEGF-B inhibited the oxidative stress-, serum deprivation-, and Bmf-induced apoptosis in the RGC5 cells.

VEGF-B inhibits axotomy-induced neuronal apoptosis and apoptotic gene expression in the retina. We next investigated the antiapoptotic effect of VEGF-B in vivo using several different animal models. First, we used the optic nerve crush (ONC) injury mouse model. In this model, it is known that ONC injury results in apoptotic death of the RGCs in the retina after the crush injury. In situ hybridization analysis showed that both VEGF-B and VEGFR-1 are highly expressed in the retina (Figure 3A). A higher level of VEGF-B expression was found in the RGCs and cells in the inner nuclear layer (Figure 3A, left, arrows). VEGFR-1 expression was mainly found in the inner plexiform, part of the inner nuclear layer, and the rod and cone layer (Figure 3A, right). Real-time PCR assay showed that both VEGF-B (Figure 3B) and VEGFR-1 (Figure 3C) were significantly upregulated in the retinae after $\mathrm{ONC}$ injury. The upregulation was seen as early as 6 hours after ONC and reached a very high level at 1 week after $\mathrm{ONC}$ injury (Figure 3, B and C; $n=4, P<0.05)$. To test whether VEGF-B can rescue RGC death, we administered human recombinant VEGF-B ${ }_{167}$ intravitreally into the mouse eyes after ONC injury and counted viable RGCs after
2 weeks. Treatment with a single dose of VEGF-B ${ }_{167}(500 \mathrm{ng} /$ eye) significantly increased the number of viable RGCs by about 1.7-fold (Figure 3, D-F; $n=8$ [vehicle] and $n=12$ [VEGF-B [V7] $_{16}$ $P<0.01)$. On the contrary, intravitreal treatment with VEGF-B neutralizing antibody (32) (5 pmol/eye) decreased the number of the viable RGCs by about 33\% (Figure 3F; $n=8, P<0.05$ ). Since it is known that VEGF-B binds to VEGFR-1 (11), we next tested whether inhibition of VEGF-B by the VEGFR-1 extracellular domain (VEGFR-1 ECD; 5 pmol/eye), acting as a ligand trap, could impair RGC survival. VEGFR-1 ECD treatment decreased the viable RGCs by about $42 \%$ (Figure 3F; $n=8, P<0.05$ ). Thus, VEGF-B is critically required for RGC survival in the injured retina.

To clarify the molecular mechanism underlying the survival effect of VEGF-B in the retina, we investigated the expression of an array of apoptotic/cell death-related genes in the retinae with or without ONC injury and with or without VEGF-B treatment 1 week after the injury. Real-time PCR analysis revealed that VEGF-B treatment (500 ng/eye) inhibited the expression of the BH3-only protein genes Noxa and Bmf, as well as the apoptotic genes Bak and $p 53$ in both normal and ONC-injured retinae (Figure 3, G-J; $n=4, P<0.05)$. It is interesting to note that Noxa expression in the retina was upregulated about 2.7 -fold 1 week after the ONC injury, 

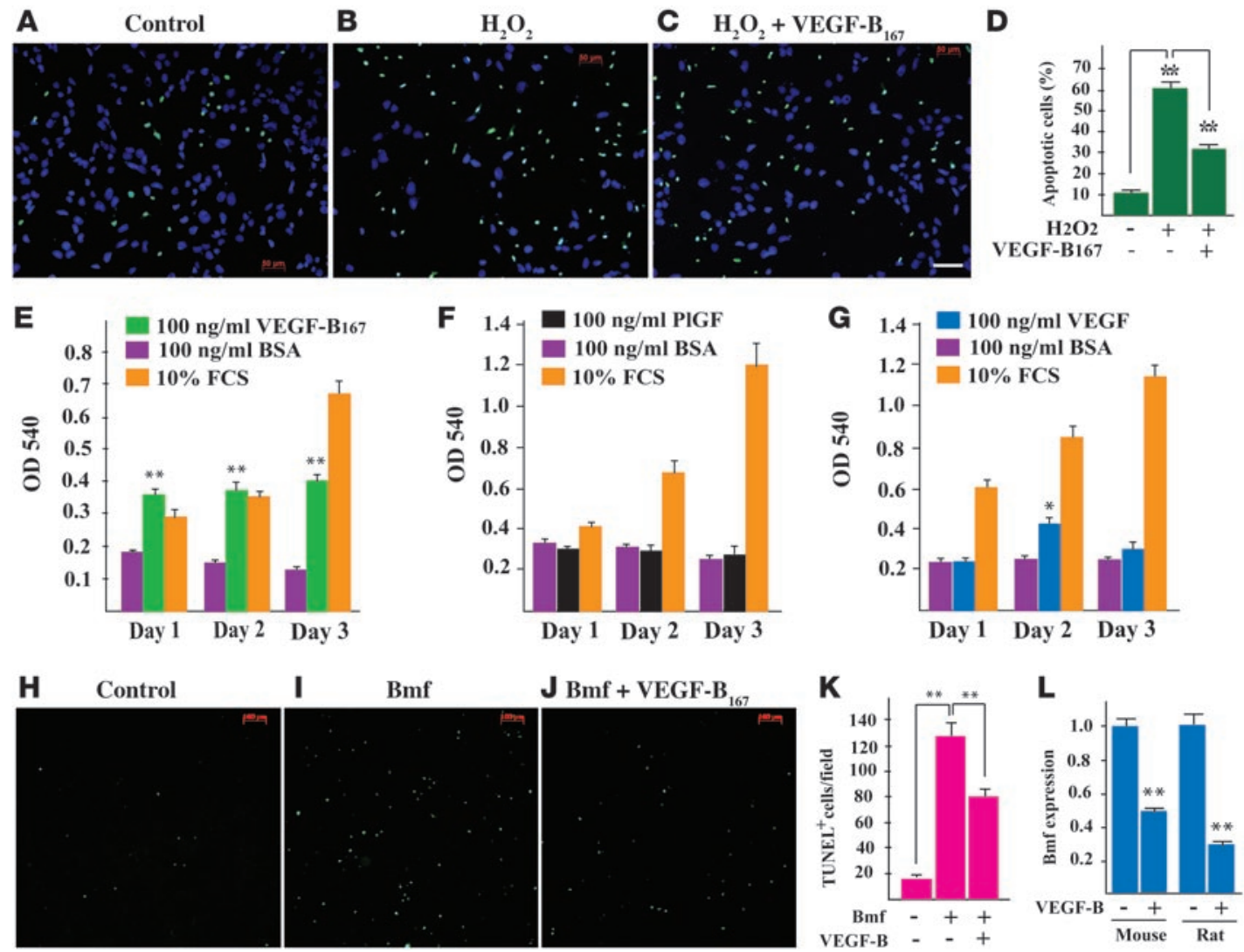

\section{Figure 2}

Survival effect of VEGF-B on the RGC5 cells. (A and B) $\mathrm{H}_{2} \mathrm{O}_{2}$ treatment led to oxidative stress-induced apoptosis in RGC5 cells according to the TUNEL assay. (C and D) VEGF- $\mathrm{B}_{167}$ treatment inhibited the $\mathrm{H}_{2} \mathrm{O}_{2}$-induced apoptosis. Scale bar: $50 \mu \mathrm{m}$. (E) VEGF-B rescued serum deprivation-induced cell death in RGC5 cells at different time points. (F) PIGF did not rescue serum deprivation-induced cell death in RGC5 cells at different time points. (G) VEGF had a weak survival effect on RGC5 cells. (H and I) Bmf overexpression in the RGC5 cells led to apoptosis according to the TUNEL assay. ( $\mathbf{J}$ and K) VEGF-B 167 treatment inhibited Bmf-induced cellular apoptosis in RGC5 cells. Scale bar: $100 \mu \mathrm{m}$. (L) VEGF-B $B_{167}$ treatment inhibited both the endogenous (rat) and exogenous (mouse) Bmf expression in RGC5 cells. ${ }^{\star} P<0.05$, ${ }^{\star \star} P<0.01$.

while no significant difference was observed with the other apoptotic genes (Figure 3, G-J). VEGF-B thus promotes RGC survival, at least partially, by inhibiting the expression of the $\mathrm{BH}$-only protein and other apoptotic genes in the retina.

VEGF-B inhibits NMDA-induced neuronal apoptosis and apoptotic/ cell death-related gene expression in the retina. In many neurodegenerative and neurologic disorders, neuronal injury and death are caused by the overstimulation of the excitatory amino acid receptors. We next tested whether VEGF-B could inhibit the excitotoxin-induced apoptosis in the retina using the NMDA injury model. We injected NMDA (20 nmol/eye) with or without VEGF-B 167 (500 ng/eye) intravitreally into mouse eyes. After 24 hours, the retinae were harvested for TUNEL staining and gene expression analysis. VEGF-B ${ }_{167}$ treatment significantly reduced the number of apoptotic cells in the RGC, the inner nuclear layer, and the outer nuclear layer (Figure 4, A-C; $n=8$, $P<0.001$ and $P<0.01)$. Real-time PCR assay revealed that VEGF-B treatment inhibited the expression of a number of the BH3-only protein genes (Bmf, Hrk, Bad, Bid, Bim), as well as other apoptotic/ cell death-related genes (TNF- $\alpha$, Trp53inp1, Casp8, Bak, Bax) in the NMDA-injured retina (Figure 4D). VEGF-B thus is a potent inhibitor of excitotoxin-induced apoptosis, at least partially, via suppression of the $\mathrm{BH} 3$-only protein and other apoptotic/cell death-related gene expression.

VEGF-B inhibits ischemia-induced neuronal apoptosis and apoptotic gene expression in the brain. It has been shown that VEGF-B deficiency led to enlarged brain infarct volume after middle cerebral artery (MCA) occlusion in mouse (33). However, the underlying molecular mechanisms remain unclear. Further, it remains unknown whether VEGF-B protein treatment could rescue ischemia-induced neuronal death in the brain. We next performed the MCA occlusion stroke model using both the VEGF-B-deficient and wild-type mice to address these questions. Immunohistochemical staining showed that both VEGF-B and VEGFR-1 are expressed in the neurons in normal brain (data not shown). VEGF-B expression was highly upregulated in the border zone of the brain after stroke (Figure 5A). VEGF-B deficiency led to about 50\% larger brain damage volume as compared with that in the wild-type mice (Table 2; $n=10, P<0.05)$. This defect was largely rescued by VEGF-B protein treatment (Table $2 ; n=9$ and $n=10, P<0.05$ ). It is interesting to note that in the wild-type mice, even though the endogenous VEGF-B was highly expressed in the brain (Figure 5A), VEGF-B protein treatment still decreased the stroke volume by about $32 \%$ (Table $2, n=8$ [vehicle] and $n=10$ [treatment], $P<0.05$; 


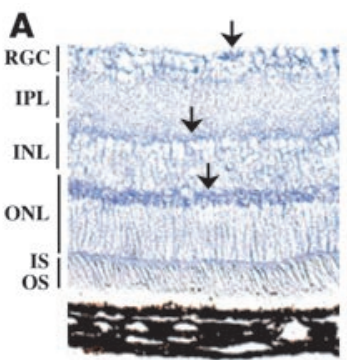

VEGF-B
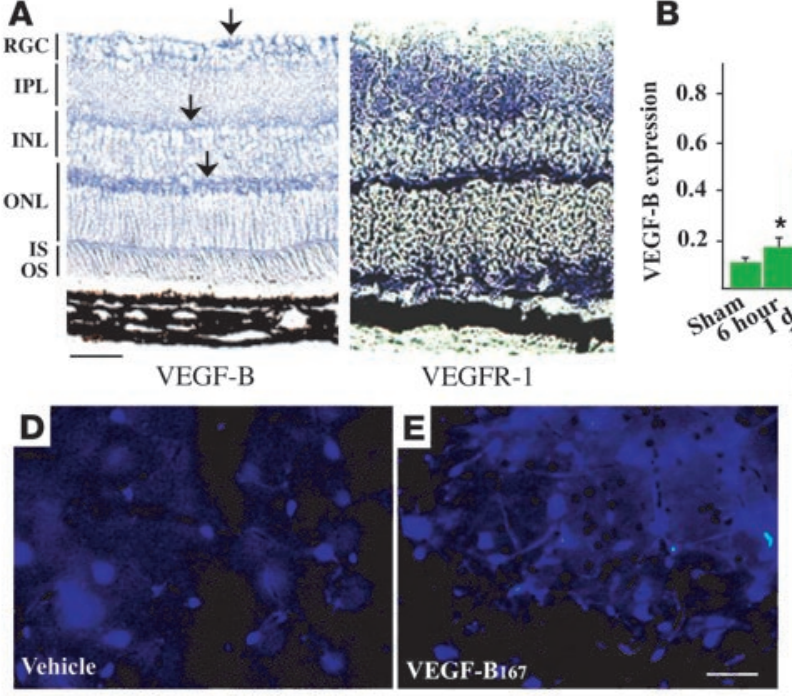

VEGFR-1

G
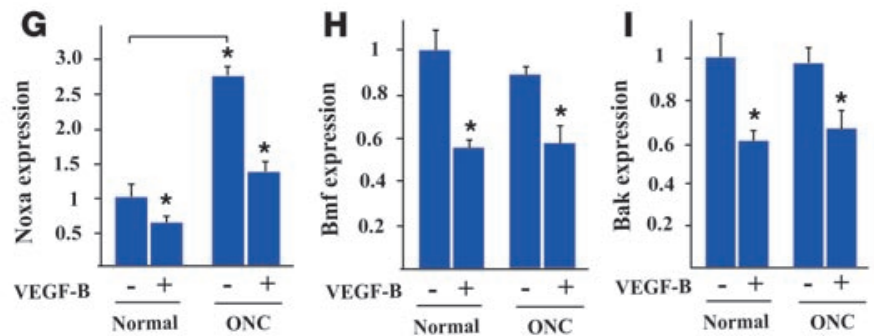

C

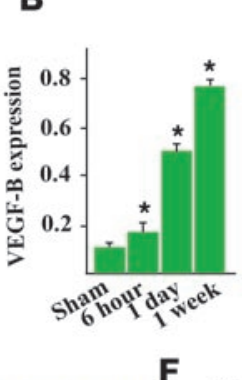

$\mathbf{F}$

(2)
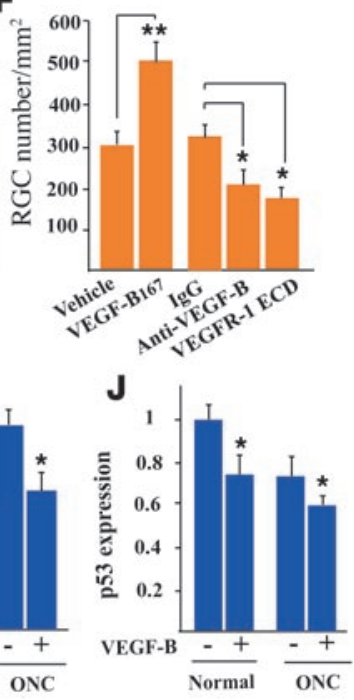

\section{Figure 3}

VEGF-B inhibits axotomy-induced apoptosis in the retina. (A) VEGF-B is highly expressed in the retina as shown by the in situ hybridization assay. A high level of VEGF-B expression was found primarily in the RGCs and the inner and outer nuclear layers (INL and ONL, arrows). VEGFR-1 expression was mainly found in the inner plexiform layer, part of the inner nuclear layer, and the inner and outer segment layers (IS, OS). Scale bar: 50 $\mu \mathrm{m}$. (B and C) Real-time PCR assay showed that VEGF-B (B) and VEGFR-1 (C) expression were upregulated in the retinae after ONC injury. The upregulation was seen as early as 6 hours after ONC and reached a high level after 1 week. (D-F) A single dose of VEGF-B ${ }_{167}$ intravitreal treatment increased the number of viable RGCs by about 1.7 -fold. VEGF-B neutralizing antibody intravitreal treatment decreased the number of viable RGCs by about $33 \%(F)$. VEGFR-1 ECD treatment decreased the number of viable RGCs by about $42 \%$ (F). Scale bar: $10 \mu \mathrm{m}$. (G-J) Real-time PCR analysis revealed that VEGF-B ${ }_{167}$ treatment inhibited the expression of the BH3-only protein genes Noxa (G) and $B m f(\mathbf{H})$, as well as Bak (I) and p53 (J) expression in both normal and ONC-injured retinae. ${ }^{*} P<0.05,{ }^{* *} P<0.01$.
Figure 5, B and C). TUNEL staining showed that VEGF-B VI67 $_{\text {treat- }}$ ment significantly decreased the number of the apoptotic cells in the border zone of the stroke, where the surviving neurons were at risk of apoptosis (number of apoptotic cells/field: $31 \pm 9$ in the control group versus $12 \pm 1$ in the VEGF-B treatment group, $n=3$, $P<0.05$; Figure 5, D and E). VEGF-B treatment thus protected neurons from apoptosis in the brain. To understand the underlying molecular mechanisms, we analyzed the expression of the BH3only protein and other apoptotic genes in the brains with MCA occlusion with or without VEGF-B treatment. VEGF-B treatment markedly downregulated the expression of the $\mathrm{BH}$-only protein genes Bmf and Hrk and the apoptotic gene Trp53inp1 according to the real-time PCR assay (Figure 5F). Thus, VEGF-B protects the neurons in the brain, at least partially, by inhibiting the expression of the $\mathrm{BH} 3$-only protein and other apoptotic/cell death-related genes.

VEGFR-1 mediates the antiapoptotic effect of VEGF-B. It is known that VEGF-B binds to VEGFR-1 (11). To verify whether

\section{Figure 4}

VEGF-B inhibits excitotoxin-induced apoptosis in the retina. (A) NMDA intravitreous treatment led to massive apoptosis in the retina as shown by TUNEL staining. (B and $\mathbf{C}$ ) VEGF-B ${ }_{167}$ treatment significantly reduced the number of apoptotic cells in all the 3 layers of the retina. Scale bar: $20 \mu \mathrm{m}$. (D) Real-time PCR assay revealed that VEGF- $B_{167}$ treatment inhibited the expression of the BH3-only protein (Bmf, Hrk, Bad, Bid, Bim) and other apoptotic/cell death-related genes in the NMDA-injured retina. ${ }^{\star \star} P<0.01,{ }^{\star \star \star} P<0.001$.
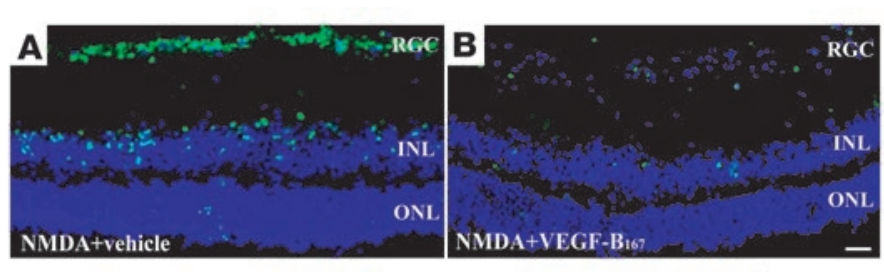

C

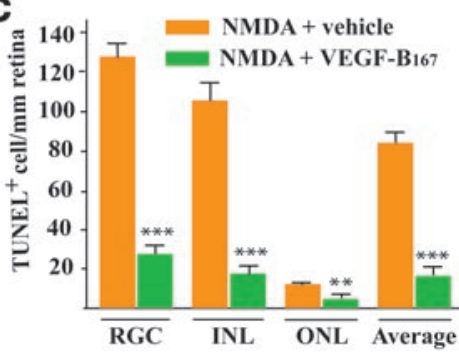

the effect of VEGF-B is mediated by VEGFR-1, we determined whether VEGFR-1 could be activated by VEGF-B in different types of cells. Using Western blot assay, VEGFR-1 was detected in the cortex neurons isolated from neonatal mice, in the bEnd. 3 cell line (transformed mouse cerebral cortex-derived endothelial cells), and in the RGC5 cells (Figure 6, A and B). Immunoprecipitation using an anti-VEGFR-1 antibody followed by Western IB assay using an anti-phosphotyrosine antibody showed that VEGF-B ${ }_{167}$ stimulation $(100 \mathrm{ng} / \mathrm{ml})$ resulted in VEGFR-1 activation in the bEnd.3 cells, cortex neurons, and the RGC5 cells (Figure 6, A and B).

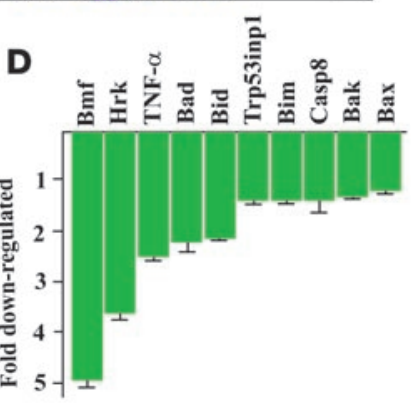



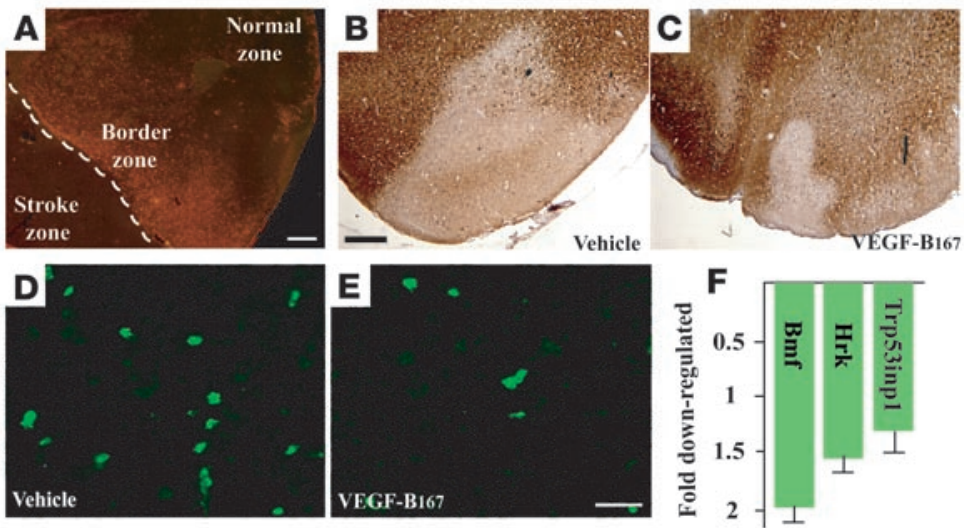

\section{Figure 5}

VEGF-B inhibits ischemia-induced neuronal apoptosis and apoptotic gene expression in the brain. (A) VEGF-B expression is upregulated in the border zone of the brain after MCA occlusion as shown by immunohistochemical staining. Scale bar: $50 \mu \mathrm{m}$. (B and C) Recombinant human VEGF-B ${ }_{167}$ protein treatment decreased brain damage volume by about $32 \%$ in the wild-type mice as shown by the MAP-2 staining. Scale bar: $100 \mu \mathrm{m}$. (D and E) VEGF-B ${ }_{167}$ treatment decreased the number of the apoptotic cells in the border zone of the stroke as shown by TUNEL staining. Scale bar: $10 \mu \mathrm{m}$. (F) VEGF-B ${ }_{167}$ treatment inhibited the expression of the $\mathrm{BH} 3$-only protein genes $\mathrm{Bmf}$ and $\mathrm{Hrk}$ and the apoptotic gene Trp53inp1 in the brain with stroke.
The cell lysate was further subjected to Western blot assay using antibodies against phosphorylated and total ERK1/2 or Akt. In both the bEnd. 3 cells and the cortex neurons, VEGF-B treatment led to ERK1/2 activation (Figure 6C). In TR-iBRB cells, VEGF-B treatment induced Akt phosphorylation (Figure 6D). However, in the other types of cells tested, VEGF-B treatment did not induce significant Akt phosphorylation (Supplemental Figure 1; supplemental material available online with this article; doi:10.1172/ JCI33673DS1), suggesting that there could be other intracellular pathways mediating the activity of VEGF-B in these cells. Thus, VEGF-B ${ }_{167}$ treatment activated VEGFR-1 followed by the activation of the ERK1/2 or Akt pathways in different types of cells.

We next tested whether VEGFR-1 blockade could abolish the inhibitory effect of VEGF-B on the expression of the apoptotic/ cell death-related genes in the RGC5 cells. VEGFR-1 neutralizing antibody treatment completely abolished the inhibitory effect of VEGF-B on the expression of Bmf, Bax, and Bik, indicating that the effect of VEGF-B indeed was mediated by VEGFR-1 (Figure 6, $\mathrm{E}-\mathrm{H} ; n=3, P<0.001$ and $P<0.05$ respectively). Moreover, VEGFR-1 neutralizing antibody treatment upregulated the expression of Bmf, Bax, and Bik (Figure 6, E-G), indicating that VEGFR-1 ligands other than VEGF-B may also contribute to the inhibition of their expression via VEGFR-1. In addition, VEGFR-1 neutralizing antibody treatment abolished the inhibitory effect of VEGF-B on the expression of Bak1 to a lesser extent (Figure $6 \mathrm{H} ; n=3$, $P<0.05)$. Thus, the inhibitory effect of VEGF-B on the expression of the BH3-only protein and other apoptotic/cell death-related genes is largely mediated by VEGFR-1. Indeed, this observation was confirmed in vivo using the ONC retinal injury model. When the VEGF-B protein was administered together with the VEGFR-1 neutralizing antibody into the mouse vitreous with ONC injury, VEGF-B-induced RGC survival was largely abolished (Figure 6, $\mathrm{I}-\mathrm{L} ; n=8, P<0.05)$. Thus, in vivo data confirmed that the antiapoptotic effect of VEGF-B is mediated by VEGFR-1.

$V E G F-B$ at a dose effective for neuron survival did not cause undesired angiogenesis in the eye. Overgrowth of blood vessels in the eye may lead to severe vision damage or loss. We therefore investigated whether intravitreal administration of VEGF-B ${ }_{167}$ could affect normal retinal vasculature and pathological angiogenesis. We sectioned the retinae with or without VEGF-B 167 treatment and stained them with isolectin B4 (IB4), which labels the vascular endothelial cells, and blood vessel density was determined in different locations of the retina (central, middle, and peripheral). No difference was observed in terms of blood vessel density and morphology 14 days after VEGF- $\mathrm{B}_{167}$ treatment (Figure 7, A-C; $n=6, P>0.05)$. Thus, VEGF-B protein treatment at a dose effective for neuron survival did not affect normal blood vessels in the retina. To investigate whether VEGF-B affects pathological angiogenesis, we used the laser-induced choroidal neovascularization (CNV) model. We administered VEGF-B ${ }_{167}$ intravitreally immediately after laser treatment. One week after VEGF-B ${ }_{167}$ treatment, when $C N V$ reaches the peak level, the eyes were harvested and the $\mathrm{CNV}$ area measured after IB4 staining. No difference was observed between the VEGF- $B_{167}$-treated and the control groups (Figure 7, $\mathrm{D}-\mathrm{F} ; n=8, P>0.05)$. Thus, VEGF-B protein treatment at a dose effective for neuron survival did not affect pathological CNV. To test whether VEGF-B is involved in blood vessel permeability, we performed the modified Miles assay using the VEGF-B-deficient and wild-type mice. No difference was found between the 2 groups $\left(\mathrm{OD}_{620}: 0.17 \pm 0.01\right.$ in VEGF-B-deficient and $0.19 \pm 0.02$ in wildtype mice, $n=10,12, P>0.05)$. Thus, at a dose effective for neuron survival, VEGF-B treatment did not seem to affect normal or pathological angiogenesis, and VEGF-B deficiency did not affect blood vessel permeability according to the modified Miles assay. However, our data do not exclude the possibility that VEGF-B in other conditions may affect the blood vessel system. Further studies are needed to look into this.

\section{Discussion}

VEGF-B was discovered many years ago and has a high sequence homology to the other VEGF family members. However, the function of VEGF-B is still a debatable issue. We reveal here for the first time to our knowledge a novel function of VEGF-B as a potent apoptosis inhibitor. VEGF-B treatment rescues neurons

\section{Table 2}

VEGF-B treatment reduces brain damage volume in both VEGF-B-deficient and wild-type mice

\begin{tabular}{lc}
\hline & Stroke volume $\left(\mathrm{mm}^{3}\right)$ \\
VEGF-B-KO & $18.9 \pm 1.9$ \\
WT & $12.8 \pm 1.8$ \\
VEGF-B-KO + VEGF-B & $10.7 \pm 2$ \\
VEGF-B-KO + vehicle & $15.9 \pm 1.7$ \\
WT + VEGF-B & $7.7 \pm 1.3$ \\
WT + vehicle & $11.3 \pm 1$ \\
\hline
\end{tabular}



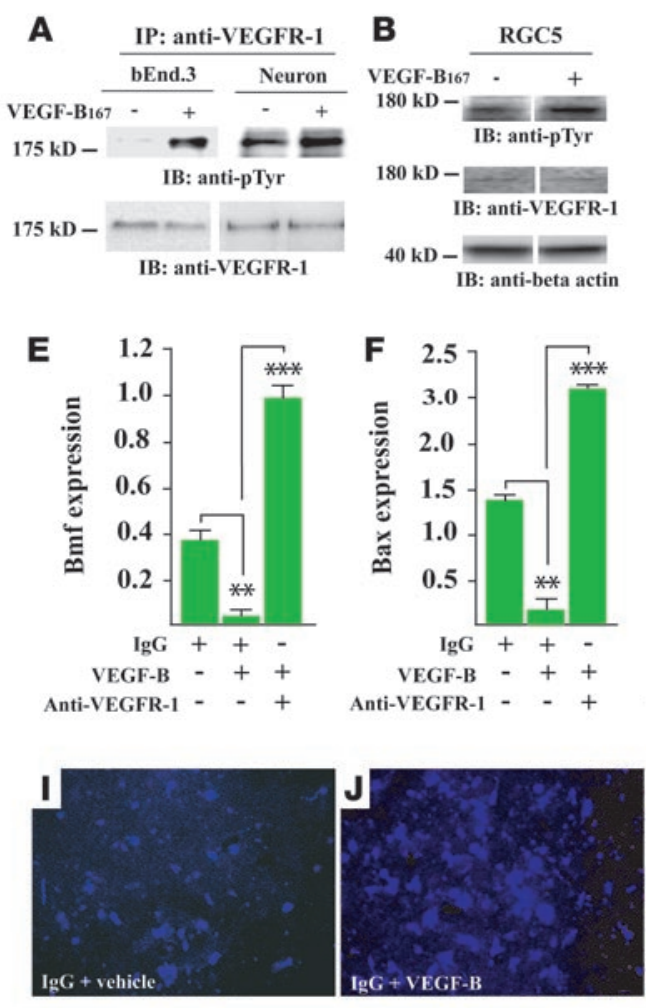
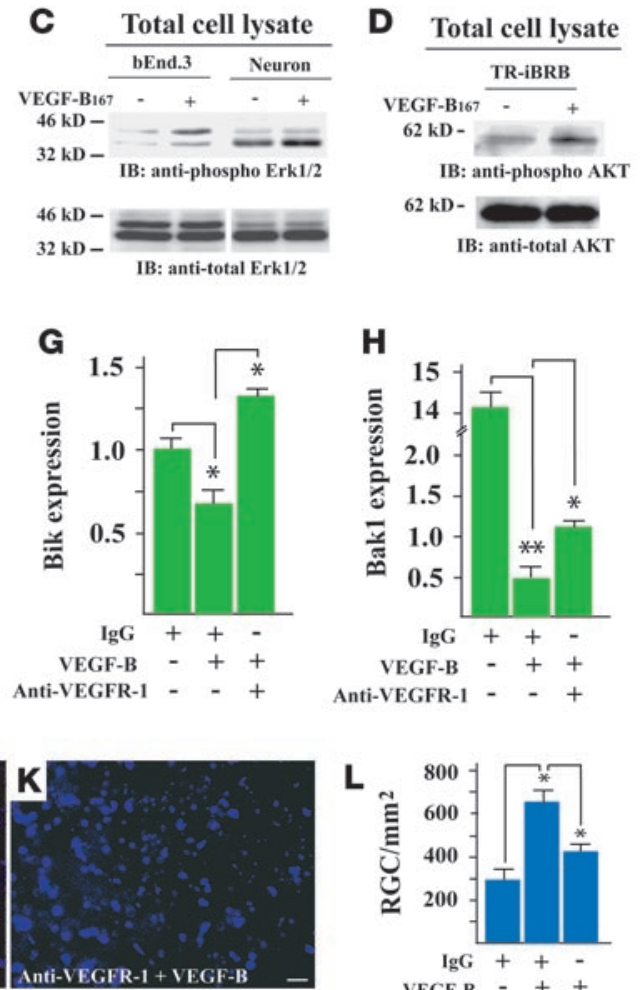

H
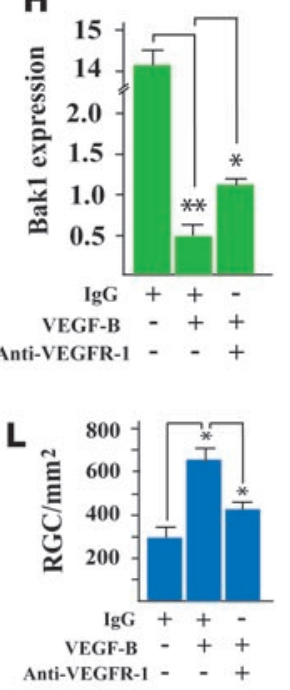

\section{Figure 6}

VEGFR-1 mediates the effect of VEGF-B. (A) VEGF-B ${ }_{167}$ stimulation resulted in VEGFR-1 activation in the bEnd. 3 cells and the cortex neurons using the immunoprecipitation assay and anti-VEGFR-1 antibody, followed by the Western blot assay using anti-phosphotyrosine antibody (anti-pTyr). VEGFR-1 was detected in bEnd. 3 cells and cortex neurons using Western blot assay. (B) VEGF-B 167 induced VEGFR-1 activation in RGC5 cells using Western blot assay and antibodies against phosphotyrosine and VEGFR-1. (C) VEGF-B $B_{167}$ treatment led to ERK1/2 activation in both bEnd.3 cells and cortex neurons using Western blot assay and antibodies against phosphorylated and total ERK1/2. (D) VEGF-B treatment induced Akt phosphorylation in TR-iBRB cells using Western blot assay and antibodies against phosphorylated and total Akt. (E-H) VEGFR-1 neutralizing antibody treatment abolished to various degrees the inhibitory effect of VEGF-B on the expression of $\operatorname{Bmf}(\mathbf{E}), \operatorname{Bax}(\mathbf{F}), \operatorname{Bik}(\mathbf{G})$, and Bak1 (H) in RGC5 cells. (I, J, and L) VEGF-B protein treatment increased RGC survival in the ONC-injured retina. Scale bar: $20 \mu \mathrm{m}$. (I, K, and L) VEGFR-1 neutralizing antibody treatment largely abolished the VEGF-B-induced RGC survival in the ONC-injured retina. ${ }^{\star} P<0.05,{ }^{* \star} P<0.01,{ }^{* \star *} P<0.001$.

from apoptosis in both the retina and brain. Mechanistically, we demonstrate that VEGF-B inhibits the expression of the BH3-only protein and other apoptotic/cell death-related genes via VEGFR-1. It is worth noting that the survival effect of VEGF-B is not associated with undesired angiogenesis at a dose effective for neuron survival. VEGF-B thus appears to be the first member of the VEGF family that has a potent antiapoptotic effect while lacking a general angiogenic activity.

Even though VEGF has also been shown to be a potent neuroprotective factor (34), the therapeutic potential of its neuroprotective effect is limited because of its potent and general angiogenic and permeability-promoting effect. In contrast to the other VEGF family members, VEGF-B does not have a general effect on angiogenesis and blood vessel permeability. This nature of VEGF-B is of particular importance for the potential clinical usage of VEGF-B as a neuroprotective factor. VEGF-B thus has an unusual safety profile, with minimal side effects, as a survival molecule. It may therefore be used to treat a broad array of neurodegenerative dis- trast to VEGF, which is a potent stimulator for vascular endothelial cell proliferation and migration, VEGF-B has little effect on endothelial cell proliferation and migration (data not shown) (9). Several studies have reported an angiogenic activity of VEGF-B (24-26). However, this has not been observed by other investigators $(16,18,20,21,27)$. Thus, studies on VEGF-B have led to debatable and inconsistent results, and the function of VEGF-B remains elusive.

In this study, we demonstrated that VEGF-B signaling is critical for cell survival. Using a genome-wide gene profiling approach, we revealed that the genes most significantly downregulated by VEGF-B are the genes that are critically involved in the apoptosis/ cell death-related pathways. We validated our results both in vitro and in vivo using multiple cell lines and animal models. Among the genes downregulated by VEGF-B, the BH3-only proteins, which are critical regulators in initiating apoptosis, were consistently inhibited by VEGF-B in different experimental settings. The BH3-only proteins are therefore the main targets suppressed by VEGF-B, suggesting a major role of VEGF-B in inhibiting apoptosis. Indeed, 

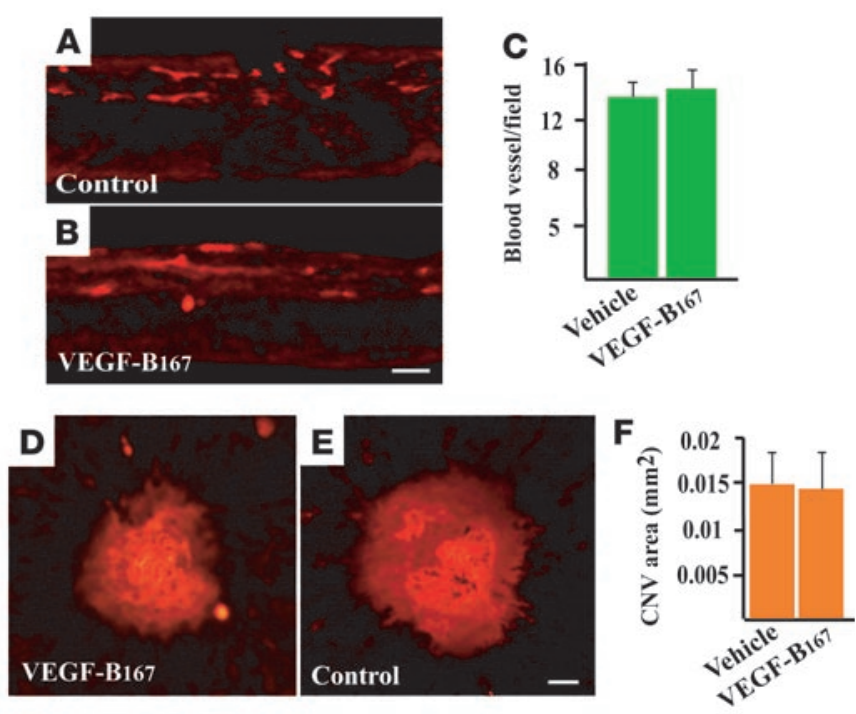

\section{Figure 7}

VEGF-B does not affect retinal angiogenesis after intravitreous injection. (A-C) VEGF-B ${ }_{167}$ treatment did not affect blood vessel density and morphology in the retina using IB4 staining. Scale bar: $50 \mu \mathrm{m}$. (D-F) VEGF-B ${ }_{167}$ intravitreous administration after laser treatment did not change the CNV area as shown by IB4 staining. Scale bar: $50 \mu \mathrm{m}$.

using different animal models including the ONC injury and the NMDA-induced retinal neuron apoptosis models, as well as the acute ischemia-induced brain neuron apoptosis model, we confirmed that VEGF-B inhibits apoptosis and promotes neuronal survival in both the retina and brain, in conditions of axotomy-, neurotoxin-, and the ischemia-induced neuronal death, at least partially, by inhibiting the expression of the BH3-only protein and other apoptotic/cell death-related genes. The $\mathrm{BH} 3$-only proteins are the key upstream regulators in initiating apoptosis. Their activities are mainly regulated at the transcription level. By inhibiting the expression of the BH3-only protein family members and other apoptotic/cell death-related genes, VEGF-B keeps apoptosis in check at an early stage. VEGF-B thus may have an effect in normal tissues similar to that of angiopoietin- 1 in endothelial cells - to stabilize and protect them against apoptosis.

Anti-VEGF therapy has been proven to be beneficial for patients with cancer or ocular neovascular diseases. However, it is known that systemic anti-VEGF treatment may lead to thromboembolic events, such as stroke, and increase the risk of neurodegeneration (40). In the eye, long-term VEGF targeting may impose a risk of ischemia and lead subsequently to neuronal apoptosis (41). Due to its potent antiapoptotic nature, VEGF-B may potentially be used clinically as an adjunct to the current anti-VEGF therapies to provide a balance between preventing neuronal apoptosis and inhibiting angiogenesis.

VEGF-B binds to VEGFR-1 and NP-1 $(10,11)$. VEGFR-1 is highly expressed in the retina and is critically required for the maintenance and survival of the retinal vasculature (42). VEGFR-1 is expressed in the brain and upregulated after brain ischemia (43), suggesting a role of VEGFR-1 in neuronal survival. Indeed, the survival effect of VEGFR-1 has been shown in numerous cell types (44-46). In this study, VEGFR-1 ECD treatment decreased the viable RGCs by about $42 \%$ in the ONC injury model, suggesting the critical role of the VEGFR-1 ligands in RGC survival. Indeed, VEGF-B stimulation led to VEGFR-1 and ERK MAPK activation in RGC5, bEnd.3, and cortex neuronal cells. Furthermore, VEGFR-1 neutralizing antibody largely abolished the inhibitory effect of VEGF-B on the expression of the apoptotic/cell death-related genes in the RGC5 cells in vitro and decreased VEGF-B-dependent RGC survival in the retina in vivo. Thus, the antiapoptotic/survival effect of VEGF-B is mainly mediated by VEGFR-1. The other receptor used by VEGF-B, NP-1, is also expressed by neurons in the retina and brain $(47,48)$ and is upregulated after focal cerebral ischemia (48). NP-1 has been shown to promote the survival of various types of cells (49). NP-1 could therefore also be involved in mediating the survival effect of VEGF-B. Further studies are needed to verify this possibility.

In summary, using multiple approaches involving the genomewide gene profiling assay, cultured cells, and animal models, we show for the first time to our knowledge that VEGF-B is a potent apoptosis inhibitor. VEGF-B rescues neurons from apoptosis in both the retina and brain without causing undesired angiogenesis. Moreover, the survival effect of VEGF-B is achieved by inhibiting the expression of the $\mathrm{BH} 3$-only protein and other apoptotic/cell death-related genes via VEGFR-1. VEGF-B appears to be the first member of the VEGF family that has a potent antiapoptotic effect but lacks general angiogenic activity. VEGF-B thus could potentially provide a new therapeutic option for treating different types of neural degenerative diseases.

\section{Methods}

Microarray analysis. Mouse aortic artery SMCs were isolated and cultured as described previously (50). SMCs were grown to $80 \%$ confluence and synchronized by overnight serum starvation followed by stimulation with human VEGF-B ${ }_{167}(100 \mathrm{ng} / \mathrm{ml})$ for 6 hours in a hypoxic ( $1 \%$ oxygen) condition. Total RNA was isolated using the TRIzol reagent (Invitrogen) followed by RNeasy Mini Kit (QIAGEN) purification according to the manufacturer's instructions. Total RNA $(10 \mu \mathrm{g})$ was subjected to cDNA preparations using Cy3-dUTP or Cy5-dUTP (Amersham Biosciences) and an oligo-dT primer. Microarray assay was performed using the Whole Mouse Genome Oligo Microarray (Agilent Technologies). This array is composed of 41,534 (60-mer) oligonucleotide probes representing more than 41,000 mouse genes and transcripts compiled from a broad sequence collection. For the first slide, the VEGF-B ${ }_{167}$-treated cDNA was labeled with the Cy3 fluorescent dye, and the control cDNA was labeled with the Cy 5 fluorescent dye. For the second slide, the VEGF-B ${ }_{167}$-treated cDNA was labeled with the Cy5 fluorescent dye, and the control cDNA was labeled with the Cy3 fluorescent dye. The whole experiment, including the treatment of the SMCs with VEGF- B $_{167}$, was repeated independently twice. Thus, 4 Agilent slides were used, and 4 sets of original signals were obtained with each RNA sample. Two-tailed Student's $t$ test was used for the statistical analysis of the expression data. The threshold of differential gene expression was set at greater than 2-fold upregulation or $50 \%$ downregulation of the genes. Functional grouping of the differentially expressed genes was performed using several different tools, including WebGestalt (http://bioinfo.vanderbilt.edu/webgestalt) and Ingenuity Pathways Analysis (https://analysis.ingenuity.com/pa/login/login.jsp).

Cell culture, cell viability, Bmfoverexpression, and real-time PCR assay. Cells of the rat RGC-derived cell line RGC5, the immortalized rat retinal pericyte cell line (TR-rPCT), and the immortalized rat retinal Müller cell line (TR-MUL) were cultured in DMEM containing 10\% FBS and 1\% penicillin/streptomycin at $33^{\circ} \mathrm{C}$ with $5 \% \mathrm{CO}_{2}$. The immortalized rat retinal capillary endothelial cell line (TR-iBRB) and the bEnd. 3 cell line derived from the transformed 
mouse cerebral cortex endothelial cells were cultured in DMEM supplemented with endothelial cell growth factors (1-033484; Roche Diagnostics). For the viability assay, RGC5 cells were starved in serum-free medium for 3 days. The cells were then seeded in 96-well tissue culture plate (BD Biosciences) and allowed to attach for 24 hours in serum-free medium. The medium was then replaced with $100 \mu \mathrm{l}$ of serum-free medium supplemented with recombinant human VEGF-B 167 protein $(100 \mathrm{ng} / \mathrm{ml})$. Serum-free medium with $100 \mathrm{ng} / \mathrm{ml} \mathrm{BSA}$ was used as a negative control. Cell culture medium with $10 \%$ FBS was used as a positive control. All the experiments were performed in triplicate. Cell viability was evaluated using the MTT method according to the manufacturer's protocol (MTT Cell Viability Assay Kit; Invitrogen) at different time points. After lysis of the cells, the formazan dye was quantified by measuring the absorbance at $540 \mathrm{~nm}$ using a photometer (Multiscan EX Microplate Photometer; Thermo Electron Corp). The mouse Bmf expression construct was a kind gift from Andreas Strasser at The Walter and Eliza Hall Institute of Medical Research, Parkville, Victoria, Australia. The DNA plasmid was transfected into the RGC5 cells using the FuGENE Transfection Reagent (Roche Diagnostics) according to the manufacturer's instructions. Apoptosis of the transfected cells was analyzed 2 days after transfection. For VEGFR-1 neutralizing antibody (R\&D Systems) treatment, the RGC5 cells were treated with the antibody at 500 $\mathrm{ng} / \mathrm{ml}$ overnight, followed by VEGF-B recombinant protein treatment at $100 \mathrm{ng} / \mathrm{ml}$ for 6 hours. For the real-time PCR assay, total RNA was isolated using the RNeasy Mini-kit (QIAGEN) according to the manufacturer's instructions. Three micrograms of total RNA was used for CDNA synthesis using the SuperMix Kit (Invitrogen) and used for the real-time PCR reaction using an ABI Prism 7500 HT Sequence Detection System (Applied Biosystems). All experiments were performed in triplicate and repeated at least twice. Primers used are listed in Supplemental Table 1.

Neonatal neuron isolation and survival assay. For neonatal neuron isolation, cerebral cortex from 1-day-old neonatal mice was collected in Neurobasal A media (Invitrogen) with $0.1 \%$ EDTA and digested with trypsin (Invitrogen) for 30 minutes at $37^{\circ} \mathrm{C}$, followed by density gradient centrifugation with $7.0 \%, 9.4 \%, 11.9 \%, 16.4 \%$ of Optiprep (AXIS-SHIELD). Neurons positioned between the second and third layers were collected and cultured in Neurobasal A for subsequent analysis. Experiments were performed within 10 days of culture. For neuronal survival assay, neurons were washed 2 times with prewarmed DMEM without glucose (Invitrogen) and then cultured in DMEM with or without $45 \mathrm{mM}$ glucose. Complete Neurobasal A medium was used as a control for $100 \%$ survival rate. For hypoxia, neurons were cultured in the Aeropack Kenki system (Mitsubishi Gas) at $37^{\circ} \mathrm{C}$. After 6 hours, media were harvested and, after the addition of $500 \mu \mathrm{l}$ of $2 \%$ Triton $\mathrm{X}-100$, kept for 15 minutes at room temperature. Lactate dehydrogenase (LDH) activity was measured in the media and cell lysate. The ratio of the $\mathrm{LDH}$ activity from the cell lysate (viable cells) to the sum of the total LDH activity (from the lysate and the medium) was used as a survival index. Experiments were performed in triplicate and were repeated at least twice.

ONC injury mouse model. All animal experiments were approved by the Animal Care and Use Committee (ACUC) at the National Eye Institute/ $\mathrm{NIH}$ and were performed according to NIH guidelines and regulations on animal studies. Female mice ( 8 weeks old) were deeply anesthetized by intraperitoneal injection of xylazine $(14 \mathrm{mg} / \mathrm{kg})$ and ketamine $(60 \mathrm{mg} / \mathrm{kg})$ and placed in a small stereotactic instrument. The skull was exposed and kept dry and clean using $3 \%$ hydrogen peroxide. The bregma was identified and marked. A hole was drilled above the superior colliculus of each hemisphere. Using a stereotactic measuring device and a Hamilton injector, the mice were injected with FluoroGold in the superior colliculus of each hemisphere at a depth of $1.6 \mathrm{~mm}$ from the bony surface of the brain, and the incision sutured. Three days after the tracer dye application, the right optic nerve of each mouse was subjected to a crush injury to cause primary dam- age to the axons. Using a binocular operating microscope, the conjunctiva of the right eye was incised. The orbital muscles were teased and deflected aside to expose the optic nerve at its exit from the globe. With the aid of the cross-action forceps, the optic nerve was subjected to a severe crush injury 1-2 mm from the eyeball. For VEGF-B treatment, under a dissecting microscope, using a 33-gauge needle, 1-2 $\mu$ l of VEGF-B solution (500 ng) with or without VEGFR-1 neutralizing antibody (20 pmol/eye; R\&D Systems) was injected into the vitreous chamber in one eye. After 2 weeks, mice were deeply anesthetized and eyes collected for analysis. Under a fluorescence microscope, 8 pictures from the central and peripheral retina respectively were taken, resulting in 16 pictures per retina using AxioVision software (Carl Zeiss Inc.), and the viable RGCs counted. The average density of the viable RGCs was calculated from the 16 pictures.

NMDA retina injury model. Eight-week-old female mice were anesthetized as described above. NMDA ( $20 \mathrm{nmol}$ in $0.5 \mu \mathrm{l}$, together with $500 \mathrm{ng}$ VEGF-B 167 protein or vehicle in $1 \mu \mathrm{l}$ ) was injected into the eye intravitreally. Intravitreal injection was performed using a 33-gauge needle from $0.5 \mathrm{~mm}$ behind the limbus in the temporal region of the globe at a $55^{\circ}$ angle to the equator. Mice with vitreous hemorrhage, retinal detachment, or lens trauma caused by the injection were excluded from the assay. Retinae were harvested 24 hours after the injection and embedded in OCT and sectioned throughout the whole eye for TUNEL staining. For quantitative analysis, 8 microscopic fields were photographed using AxioVision software (Carl Zeiss Inc.) from 8 sections of the most severely injured parts of the retina, TUNEL-positive nuclei were counted, and mean value was calculated.

Cerebral ischemic stroke model. Focal cerebral ischemia was produced by permanent occlusion of the MCA as described previously (51). VEGF-Bdeficient mice were described previously (16). Littermates from the mice that were backcrossed onto the C57BL/ 6 background for more than 6 generations were used for the experiments. Briefly, 8-week-old male mice were anesthetized, and a U-shape incision was made between the left ear and the left eye. A small opening (1-2 $\mathrm{mm}$ in diameter) was made in the region over the MCA with a handheld drill. The MCA was ligated and transected distally to the ligation point. After 24 hours, the mice were killed with an overdose of Nembutal (500 mg/kg; Abbott Laboratories) and perfused with $1 \%$ PFA. The brains were removed, postfixed in $1 \%$ PFA overnight, and subjected to MAP-2 immunohistological staining. The infarct volume was defined as the sum of the MAP-2 negative areas of the sections multiplied by their thickness using AxioVision software (Carl Zeiss Inc.). For VEGF-B protein treatment, a skin incision was made at the top of the head of the anesthetized mouse. Four small openings ( $1 \mathrm{~mm}$ in diameter) were made in the skull at $-1,0,1,2 \mathrm{~mm}$ from the bregma to the lambda and $1 \mathrm{~mm}$ left from the bregma. A fine needle (33 gauge) was inserted to the depth of $1.2 \mathrm{~mm}$ from the skull surface, and $1 \mu \mathrm{l}$ of $0.55 \mu \mathrm{g} / \mu \mathrm{l}$ recombinant human VEGF- $B_{167}$ was injected over 5 minutes into each opening and the skin sutured. MCA ligation was then performed as described above.

Laser-induced CNV model and Miles assay. Ten-week-old female mice were anesthetized and pupils dilated. Mice were positioned on a rack connected to a slit lamp delivery system. Four photocoagulation spots were made (75- $\mu \mathrm{m}$ spot size, $75 \mathrm{~ms}, 90 \mathrm{~mW}$ power; Oculight Infrared Laser System 810 $\mathrm{nm}$; IRIDEX Corp.) in the area surrounding the optic nerve in each eye. The sites were visualized through a handheld contact lens and a viscous surface lubricant. Only laser-induced burns with a bubble formation were included in the study. The mice were given lubricant ophthalmic ointment after laser treatment. Seven days after laser treatment, the eyes were removed and fixed, the retina dissected, and choroids isolated and stained with IB4 conjugated with Alexa Fluor 568 (Invitrogen). The eyecups were flat-mounted in Aquamount with the sclera facing down and the total neovascular area measured using AxioVision software (Carl Zeiss Inc.) and the mean value per burn presented for each eye. Miles assay was performed as described previously (24). 
TUNEL staining and in situ hybridization. TUNEL assay was performed according to the manufacturer's protocol (Roche Diagnostics). In situ hybridization was performed as described previously (52) using a mouse VEGF-B antisense riboprobe on $12-\mu \mathrm{m}$ frozen tissue sections. A sense riboprobe was used as a negative control. The riboprobes were prepared using the T7 and T3 RNA polymerases and the digocigenin-11-UTP (Roche Diagnostics) according to the manufacturer's instructions.

Cloning and expression of buman VEGFR-1 ECD. Human VEGFR-1 (NM_002019) cDNA plasmid was obtained from Nader Rahimi (Boston University School of Medicine, Boston, Massachusetts, USA). The 2,212-bp (125-861 aa) VEGFR-1 ECD was PCR amplified using the follow primers: forward 5'-CACGGATCCCACAGGATCTAGTTCAGGTTC-3' (including a BamHI site), reverse 5'-GCGAATTCTTAGTGATGGTGATGGTGATGCAGATTAGACTTGTCCGAGGT-3' (including an EcoRI site and a C-terminal $6 \times$ his tag). The PCR product was cloned into the pAcGP67A Baculovirus transfer vector $(\mathrm{BD})$ and sequencing verified. The expression vector was cotransfected with the linearized baculovirus DNA into Sf9 insect cells and the virus amplified according to the manufacturer's protocol (BD Biosciences - Pharmingen) and as described previously (53). The recombinant VEGFR-1 ECD was purified using Ni-NTA agarose according to the manufacturer's protocol (QIAGEN).

VEGFR-1, ERK1/2, and Akt expression and activation assay. A rabbit polyclonal antibody raised against aa 23-247 within the ECD of human VEGFR-1 (sc-9029; Santa Cruz Biotechnology Inc.) was used to immunoprecipitate VEGFR-1 in the cell lysate. The immunoprecipitate was analyzed by Western blotting using an anti-phosphotyrosine antibody (sc-7020; Santa Cruz Biotechnology Inc.) and a rabbit VEGFR-1 affinity-purified polyclonal antibody (sc-316; Santa Cruz Biotechnology Inc.) raised against the peptide mapping at the carboxyl terminus of human VEGFR-1. Antibodies against phosphorylated and total ERK1/2 or Akt (Cell Signaling Technology) were used in the Western blot assay to detect ERK1/2 or Akt activation.

Statistics. Two-tailed Student's $t$ test was used for statistical analysis. Difference was considered statistically significant when $P<0.05$. The data are represented as mean \pm SEM of the number of the determinations. Assays using cultured cells were performed in triplicate.

\section{Acknowledgments}

We would like to thank Neeraj Agarwal at the Department of Pathology and Anatomy, University of North Texas Health Science Center, for kindly providing the RGC5 cells; Ken-ichi Hosoya at the Faculty of Pharmaceutical Sciences, University of Toyama, for kindly providing the rat retinal cell lines; Andreas Strasser at The Walter and Eliza Hall Institute of Medical Research, for kindly providing the mouse Bmf expression plasmid; and Nader Rahimi at the Boston University School of Medicine for kindly providing the human VEGFR-1 cDNA plasmid. This research was supported in part by the National Glaucoma Research program of the American Health Assistance Foundation; the Intramural Research Program of the NIH; the National Institute on Aging; and the National Eye Institute.

Received for publication August 20, 2007, and accepted in revised form December 5, 2007.

Address correspondence to: Xuri Li, NEI/NIH, Porter Neuroscience Research Center, Building 35, 35 Convent Dr. MSC 3731, Bethesda, Maryland 20892, USA. Phone: (301) 496-4103; Fax: (301) 480-2640; E-mail: lixur@nei.nih.gov.
1. Folkman, J. 2007. Angiogenesis: an organizing principle for drug discovery? Nat. Rev. Drug Discov. 6:273-286.

2. Ferrara, N., and Kerbel, R.S. 2005. Angiogenesis as a therapeutic target. Nature. 438:967-974.

3. Carmeliet, P., and Jain, R.K. 2000. Angiogenesis in cancer and other diseases. Nature. 407:249-257.

4. Luttun, A., et al. 2002. Revascularization of ischemic tissues by PlGF treatment, and inhibition of tumor angiogenesis, arthritis and atherosclerosis by anti-Flt1. Nat. Med. 8:831-840.

5. Alitalo, K., Tammela, T., and Petrova, T.V. 2005. Lymphangiogenesis in development and human disease. Nature. 438:946-953.

6. Carmeliet, P., and Tessier-Lavigne, M. 2005. Common mechanisms of nerve and blood vessel wiring. Nature. 436:193-200.

7. Greenberg, D.A., and Jin, K. 2005. From angiogenesis to neuropathology. Nature. 438:954-959.

8. Li, X., and Eriksson, U. 2001. Novel VEGF family members: VEGF-B, VEGF-C and VEGF-D. Int. J. Biochem. Cell Biol. 33:421-426.

9. Nash, A.D., Baca, M., Wright, C., and Scotney, P.D. 2006. The biology of vascular endothelial growth factor-B (VEGF-B). Pulm. Pharmacol. Ther. 19:61-69.

10. Olofsson, B., et al. 1996. Vascular endothelial growth factor B, a novel growth factor for endothelial cells. Proc. Natl. Acad. Sci. U. S. A. 93:2576-2581.

11. Olofsson, B., et al. 1998. Vascular endothelial growth factor B (VEGF-B) binds to VEGF receptor-1 and regulates plasminogen activator activity in endothelial cells. Proc. Natl. Acad. Sci. U. S. A. 95:11709-11714.

12. Li, X., Aase, K., Li, H., von Euler, G., and Eriksson, U. 2001. Isoform-specific expression of VEGF-B in normal tissues and tumors. Growth Factors. 19:49-59.

13. Aase, K., et al. 1999. Localization of VEGF-B in the mouse embryo suggests a paracrine role of the growth factor in the developing vasculature. Dev. Dyn. 215:12-25.
14. Ferrara, N., et al. 1996. Heterozygous embryonic lethality induced by targeted inactivation of the VEGF gene. Nature. 380:439-442.

15. Carmeliet, P., et al. 1996. Abnormal blood vessel development and lethality in embryos lacking a single VEGF allele. Nature. 380:435-439.

16. Aase, K., et al. 2001. Vascular endothelial growth factor-B-deficient mice display an atrial conduction defect. Circulation. 104:358-364.

17. Bellomo, D., et al. 2000. Mice lacking the vascular endothelial growth factor-B gene (Vegfb) have smaller hearts, dysfunctional coronary vasculature, and impaired recovery from cardiac ischemia. Circ. Res. 86:E29-E35.

18. Reichelt, M., et al. 2003. Vascular endothelial growth factor-B and retinal vascular development in the mouse. Clin. Experiment. Ophthalmol. 31:61-65.

19. Louzier, V., et al. 2003. Role of VEGF-B in the lung during development of chronic hypoxic pulmonary hypertension. Am. J. Physiol. Lung Cell Mol. Physiol. 284:L926-L937.

20. Rissanen, T.T., et al. 2003. VEGF-D is the strongest angiogenic and lymphangiogenic effector among VEGFs delivered into skeletal muscle via adenoviruses. Circ. Res. 92:1098-1106.

21. Bhardwaj, S., et al. 2003. Angiogenic responses of vascular endothelial growth factors in periadventitial tissue. Hum. Gene Ther. 14:1451-1462.

22. Brkovic, A., and Sirois, M.G. 2007. Vascular permeability induced by VEGF family members in vivo: Role of endogenous PAF and NO synthesis. J. Cell. Biochem. 100:727-737.

23. Abraham, D., et al. 2002. VEGF-A and -C but not -B mediate increased vascular permeability in preserved lung grafts. Transplantation. 73:1703-1706.

24. Mould, A.W., et al. 2005. Transgenic overexpression of vascular endothelial growth factor-B isoforms by endothelial cells potentiates postnatal vessel growth in vivo and in vitro. Circ. Res. 97:e60-e70.
25. Silvestre, J.S., et al. 2003. Vascular endothelial growth factor-B promotes in vivo angiogenesis. Circ. Res. 93:114-123.

26. Wright, C.E. 2002. Effects of vascular endothelial growth factor (VEGF)A and VEGFB gene transfer on vascular reserve in a conscious rabbit hindlimb ischaemia model. Clin. Exp. Pharmacol. Physiol. 29:1035-1039.

27. Tammela, T., Enholm, B., Alitalo, K., and Paavonen, K. 2005. The biology of vascular endothelial growth factors. Cardiovasc. Res. 65:550-563.

28. Wanstall, J.C., et al. 2002. Vascular endothelial growth factor-B-deficient mice show impaired development of hypoxic pulmonary hypertension. Cardiovasc. Res. 55:361-368.

29. Puthalakath, H., et al. 2001. Bmf: a proapoptotic $\mathrm{BH} 3$-only protein regulated by interaction with the myosin $\mathrm{V}$ actin motor complex, activated by anoikis. Science. 293:1829-1832.

30. Okamura, S., et al. 2001. p53DINP1, a p53-inducible gene, regulates p53-dependent apoptosis. Mol. Cell. 8:85-94.

31. Seidler, D.G., et al. 2006. Decorin protein core inhibits in vivo cancer growth and metabolism by hindering epidermal growth factor receptor function and triggering apoptosis via caspase- 3 activation. J. Biol. Chem. 281:26408-26418.

32. Scotney, P.D., et al. 2002. Human vascular endothelial growth factor B: characterization of recombinant isoforms and generation of neutralizing monoclonal antibodies. Clin. Exp. Pharmacol. Physiol. 29:1024-1029.

33. Sun, Y., et al. 2004. Increased severity of cerebral ischemic injury in vascular endothelial growth factor-B-deficient mice. J. Cereb. Blood Flow Metab. 24:1146-1152.

34. Nishijima, K., et al. 2007. Vascular endothelial growth factor-A is a survival factor for retinal neurons and a critical neuroprotectant during the 
adaptive response to ischemic injury. Am. J. Pathol. 171:53-67.

35. Ferrara, N., et al. 1996. Heterozygous embryonic lethality induced by targeted inactivation of the VEGF gene. Nature. 380:439-442.

36. Karkkainen, M.J., et al. 2004. Vascular endothelial growth factor $\mathrm{C}$ is required for sprouting of the first lymphatic vessels from embryonic veins. Nat. Immunol. 5:74-80.

37. Dvorak, H.F., Brown, L.F., Detmar, M., and Dvorak, A.M. 1995. Vascular permeability factor/vascular endothelial growth factor, microvascular hyperpermeability, and angiogenesis. Am. J. Pathol. 146:1029-1039.

38. Carmeliet, P., et al. 2001. Synergism between vascular endothelial growth factor and placental growth factor contributes to angiogenesis and plasma extravasation in pathological conditions. Nat. Med. 7:575-583.

39. Joukov, V., et al. 1997. Proteolytic processing regulates receptor specificity and activity of VEGF-C. EMBO J. 16:3898-3911.

40. Rosenfeld, P.J., Rich, R.M., and Lalwani, G.A. 2006 Ranibizumab: Phase III clinical trial results. $O p h$ thalmol. Clin. North Am. 19:361-372.
41. Vredenburgh, J.J., et al. 2007. Phase II trial of bevacizumab and irinotecan in recurrent malignant glioma. Clin. Cancer Res. 13:1253-1259.

42. Shih, S.C., Ju, M., Liu, N., and Smith, L.E. 2003 Selective stimulation of VEGFR-1 prevents oxygeninduced retinal vascular degeneration in retinopathy of prematurity. J. Clin. Invest. 112:50-57.

43. Plate, K.H., Beck, H., Danner, S., Allegrini, P.R., and Wiessner, C. 1999. Cell type specific upregulation of vascular endothelial growth factor in an MCAocclusion model of cerebral infarct. J. Neuropathol. Exp. Neurol. 58:654-666.

44. Gerber, H.P., et al. 2002. VEGF regulates haematopoietic stem cell survival by an internal autocrine loop mechanism. Nature. 417:954-958.

45. Shih, S.C., et al. 2003. Transforming growth factor beta 1 induction of vascular endothelial growth factor receptor 1: mechanism of pericyte-induced vascular survival in vivo. Proc. Natl. Acad. Sci.U. S. A. 100:15859-15864.

46. Bates, R.C., et al. 2003. Flt-1-dependent survival characterizes the epithelial-mesenchymal transition of colonic organoids. Curr. Biol. 13:1721-1727.

47. Gariano, R.F., Hu, D., and Helms, J. 2006. Expression of angiogenesis-related genes during retinal development. Gene Expr. Patterns. 6:187-192.

48. Beck, H., et al. 2002. Cell type-specific expression of neuropilins in an MCA-occlusion model in mice suggests a potential role in post-ischemic brain remodeling. J. Neuropathol. Exp. Neurol. 61:339-350.

49. Moreno-Flores, M.T., et al. 2003. Semaphorin 3C preserves survival and induces neuritogenesis of cerebellar granule neurons in culture. J. Neurochem. 87:879-890.

50. Seidel, M.F., Simard, J.M., Hunter, S.F., and Campbell, G.A. 1991. Isolation of arteriolar microvessels and culture of smooth muscle cells from cerebral cortex of guinea pig. Cell Tissue Res. 265:579-587.

51. Nagai, N., Vanlinthout, I., and Collen, D. 1999. Comparative effects of tissue plasminogen activator, streptokinase, and staphylokinase on cerebral ischemic infarction and pulmonary clot lysis in hamster models. Circulation. 100:2541-2546.

52. Darby, I.A., Bisucci, T., Desmouliere, A., and Hewitson, T.D. 2006. In situ hybridization using cRNA probes: isotopic and nonisotopic detection methods. Methods Mol. Biol. 326:17-31.

53. Li, X., et al. 2000. PDGF-C is a new protease-activated ligand for the PDGF alpha-receptor. Nat. Cell Biol. 2:302-309. 